\title{
Assessing Hydration in Children: From Science to Practice
}

\author{
I. Guelinckx ${ }^{a}$ A.S. Frémont-Marquis ${ }^{a} \quad$ E. Eon ${ }^{b} \quad$ S.A. Kavouras ${ }^{c}$ L.E. Armstrong ${ }^{d}$ \\ ${ }^{a}$ Hydration and Health Department, Danone Research, and bSensory and Behavior Science, Danone Research, \\ Palaiseau, France; 'Department of Health, Human Performance and Recreation, University of Arkansas, \\ Fayetteville, Ark., and d Human Performance Laboratory, Department of Kinesiology, University of Connecticut, \\ Storrs, Conn., USA
}

\section{Key Words}

Hydration - Dehydration - Urine color - Biological markers .

Water · Fluid intake $\cdot$ Children · Self-assessment

\begin{abstract}
Raising children's awareness about their hydration status could be done through a noninvasive biomarker. Urine color (UC) has been validated as a biomarker of hydration in adults and children aged 8-14 years. The aim of this survey was to design and to evaluate the level of understanding and attractiveness of a self-assessment, UC-based hydration tool for children aged 6-11 years. The first phase of the survey consisted of face-to-face interviews during which 84 children identified those graphical elements necessary to understand the hydration message from 6 illustration-based designs containing the UC chart. The graphic elements selected were the basis to create 3 new designs. During the 2nd phase, the level of understanding and attractiveness of these 3 new designs was then evaluated via an online questionnaire by a total of 1,231 children in 3 countries. The design with the highest level of understanding was totally or partially understood by $76 \%$ of the participants, independent of age and gender. The levels of understanding, however, differed in the countries. In Indonesia, the levels of understanding of the 3 designs were comparable; whereas in
\end{abstract}

both France (74\%) and Mexico (78\%), significantly more participants totally and partially understood one of the 3 designs. The levels of attractiveness of the 3 designs were comparable, independent of country, age, and gender. On average, $80 \%$ of all participants liked the 3 designs a bit or a lot. Only $14 \%$ did not like the designs, and $5 \%$ of participants had no opinion regarding attractiveness. These results indicated that three out of four children like and understand the correct hydration message from a strictly illustration-based tool containing the eight-point UC scale.

(c) 2015 S. Karger AG, Basel

\section{Introduction}

The principles of the body water balance, the equilibrium between body water gain and body water losses, are the same for individuals of all ages. However, infants and children are, from a hydration physiology point of view, different from adults. Children have a higher body water content relative to body mass and, until they reach adult size, lose more water from the skin at rest and under thermoneutral conditions compared with adults [1, 2 ]. Due to these differences, children have higher water requirements per unit of body weight compared with adults [3].

\begin{tabular}{ll}
\hline KARGER 125.s. & $\begin{array}{l}\text { ( ) 2015 S. Karger AG, Basel } \\
0250-6807 / 15 / 0667-0005 \$ 39.50 / 0 \quad \text { Karger }\end{array}$ \\
$\begin{array}{l}\text { E-Mail karger@karger.com } \\
\text { www.karger.com/anm }\end{array}$ & $\begin{array}{l}\text { This is an Open Access article licensed under the terms of the } \\
\text { Creative Commons Attribution-NonCommercial 3.0 Un- } \\
\text { ported license (CC BY-NC) (www.karger.com/OA-license), } \\
\text { applicable to the online version of the article only. Distribu- } \\
\text { tion permitted for non-commercial purposes only. }\end{array}$
\end{tabular}

Isabelle Guelinckx, $\mathrm{PhD}$

Danone Nutricia Research RD 128

FR-91767 Palaiseau (France)

E-Mail isabelle.guelinckx@danone.com 
To assess whether these water requirements are met, body water gain should be evaluated. Sources of body water gain are, from largest to smallest contribution, water from fluids (drinking water and beverages of all kind), water from food, and metabolic water. Results from nutritional surveys showed that a high proportion of children and adolescents are at risk of an inadequate intake [4]. Noncompliance to the EFSA reference values for water intake ranged from $30 \%$ in Brazil, Spain, and the United Kingdom to $>80 \%$ in France, Belgium, and China among children aged 4-9. The probability of noncompliance was higher among boys and adolescents (10-17 years) than among girls and children (4-9 years) [4].

Even though no conclusion on hydration status can be drawn from these surveys, due to the lack of a biomarker and detailed data on physical activity, these results are reason for concern. The first reason of concern is the possible health impact. Even if the level of evidence is limited, a negative effect of mild dehydration on cognitive and physical performance has been demonstrated among children $[5,6]$. Besides the amount of total fluid intake, the choice of fluid type can also have a negative health consequence. Children and adolescents consuming SSBs on a daily basis are at an increased risk of becoming overweight or obese compared to non-regular consumers [7]. In contrast, the beneficial effect of increased water consumption also has been demonstrated among children $[8,9]$. The second reason for concern is the long-term behavioral effect. Habits (i.e., sugared soda consumption) are established early in life and can predict unhealthy dietary intake patterns that persist during childhood and into adolescence [10]. Therefore, raising awareness among children regarding the importance of a healthy and adequate fluid intake seems, appropriate. Raising awareness could be done through the evaluation of someone's daily total fluid intake; however, this is a burdensome task. A noninvasive biomarker that can be effectively used by a child during daily activities could address this issue, if this biomarker assesses hydration status in an accurate and reliable way [11]. Urine color has been identified to fit these criteria, and it was validated among adults approximately 20 years ago [12]. Recently, urine color has also been tested as a hydration biomarker among children. Urine color displayed in a clinical trial a positive relationship with urine osmolality [13]. Moreover, urine color measured on a 24 -h, a first-morning, and a before-lunch spot urine sample of 2010 children was shown to have a good diagnostic ability with good sensitivity (92 to 98\%) for detecting hypohydration (Uosm $\geq$ $800 \mathrm{mmol} / \mathrm{kg}$ ). This study also reported that children were able to accurately self-assess the urine color in 67 and $74 \%$ of, the morning and the noon samples [13]. These data clearly support and expand the applicability of the urine color chart in children. If the urine color chart is distributed to children or parents, the tool containing the urine color chart ideally is self-explanatory and the child is enthusiastic to use the tool. The aim of the survey presented in this paper was therefore to design a hydration self-assessment tool that is based on the urine color chart and is universally understood by children aged 6-11.

\section{Method}

Different designs of the urine color tool were created by a professional communication agency. All designs were strictly illustration based and contained the urine color chart combined with graphical elements such as drops, a boy, a clock, or a stock of water.

These artworks were tested in a two-phase protocol (figure 1). The aim of phase 1 was to select those graphic elements necessary for children to understand the hydration message, with the purpose of creating a new design from 6 original formats. The graphic elements selected were the basis to create 3 new designs. The level of understanding and attractiveness of these 3 new artworks were then evaluated in phase 2 . The final aim of phase 2 was to select one design with the highest level of understanding and attractiveness for children. Copies of this final artwork can be obtained at www.h4hinitiative.com.

For phase 1 and phase 2, respectively, 84 children and 1231 children in total were recruited. During recruitment, an equal distribution between sex and age group (6-8 years and 9-11 years) was taken into account. Since a universal understanding of the tool was sought, recruitment took place in 3 countries (France, Mexico and Indonesia) from different continents. The parents of all participants were informed about the aim of the survey and gave voluntary consent for their child to participate in the survey.

During phase 1, a $30 \mathrm{~min}$ face-to-face, in-depth interview between the parent and the child led to the identification of the graphical elements necessary for understanding. Through 8 open questions, the parents collected the feedback of the child on the tool. An example of open question is: 'Let your child tell the story he/she understood, as if he/she was telling this story to his/her best friend.' Additional multiple choice questions were asked to understand more in depth the reasons of the unattractiveness and misunderstanding of certain graphical elements. An example of this type of question was: 'What do you think the colors in the boxes represent?'

The questionnaire for phase 2 was an online questionnaire. A first part of the questionnaire included 5 open questions aimed at evaluating spontaneous understanding of the tool, the urine colors, and the graphical elements. Examples of these questions are: 'What do you think the colors in the boxes represent?' or 'Imagine your pee is that color, what does that mean according to your understanding of this tool?' All responses were translated through a 'verbatim' codification into the following categories: understand totally (both urine color and hydration/drinking water were quoted), understand partially (either urine color, either drinking water/hydration was quoted), misunderstanding (interpretations such as heat or skin color were quoted), and don't know (child unable to describe any message of the tool). A second part of the questionnaire
6

Ann Nutr Metab 2015;66(suppl 3):5-9 DOI: $10.1159 / 000381814$
Guelinckx/Frémont-Marquis/Eon/

Kavouras/Armstrong 


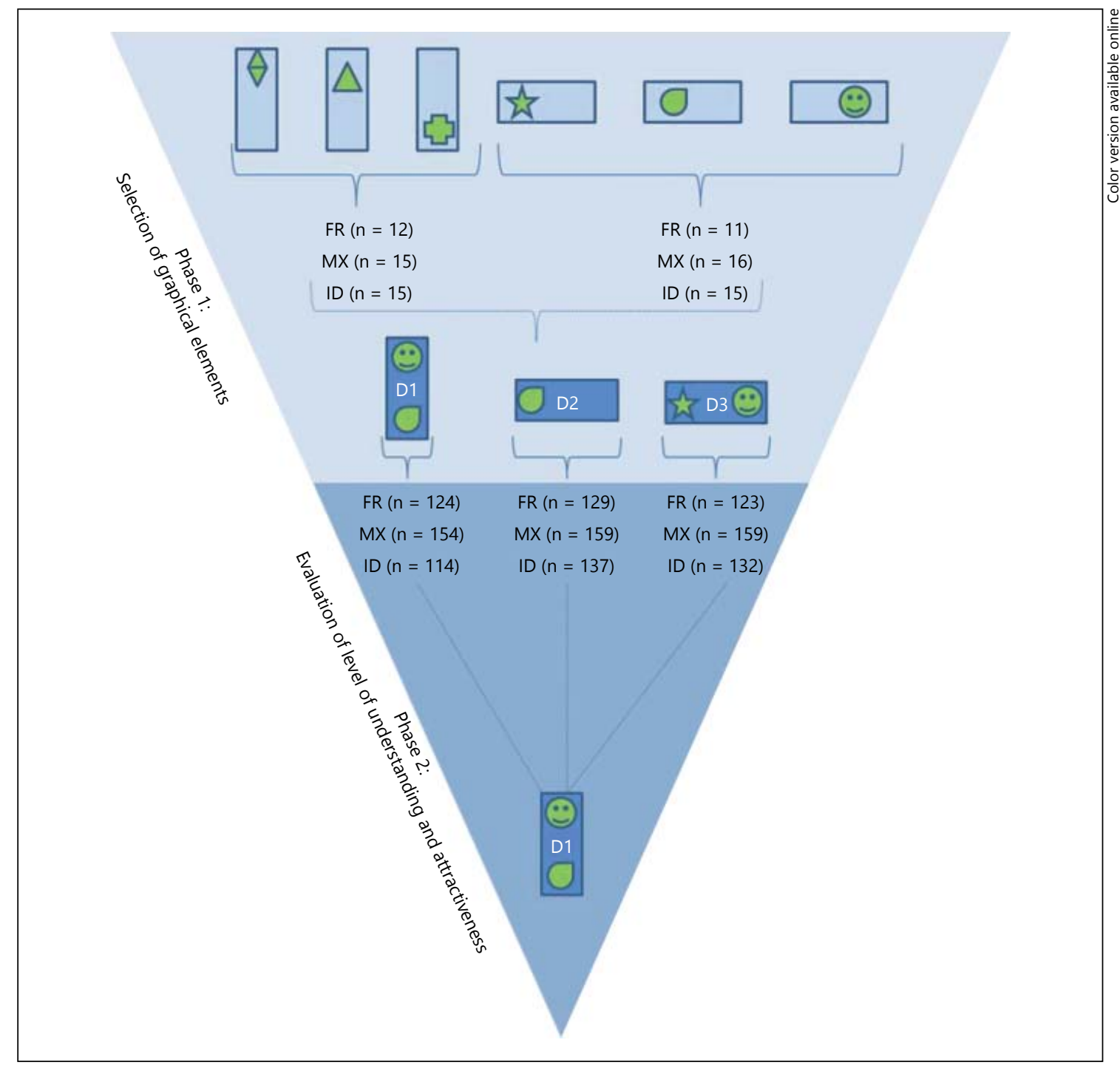

Fig. 1. Survey protocol to identify the graphic elements (phase 1) and test the comprehension and attractiveness of a urine color tool (phase 2) for children. The graphic elements and the tool are schematically represented in this figure; the final tool can be viewed at www.h4hinitiative.com.

contained statements related to the message of the tool; the children evaluated the statement on a 4 -point Likert scale ranging from 'completely false' to 'completely true.' The level of attractiveness of the tool was also assessed via a 5 -point Likert scale, with questions ranging from 'I like it very much' to 'I don't like it at all.'

\section{Results}

During the phase 1 interviews, the following graphic elements were retained as being necessary for global understanding of the tool: the urine color scale, the red-orange- green color codes, the water drops, the illustration with a child drinking water, and the illustration of the toilets.

Design D1 resulted in the highest level of spontaneous understanding in all 3 countries: $60 \%$ and $16 \%$ of the participants' responses fell into the 'partially' or 'totally' understood categories (figure 2). The illustration of the child along with the red, orange, and green color codes greatly helped in understanding the importance of drinking water (examples: 'when the boy does not drink, he is dehydrated' or 'the more you drink, the less dark your urine, is'). Age and gender did not significantly affect the level of understanding (data not shown), but the country of residence 


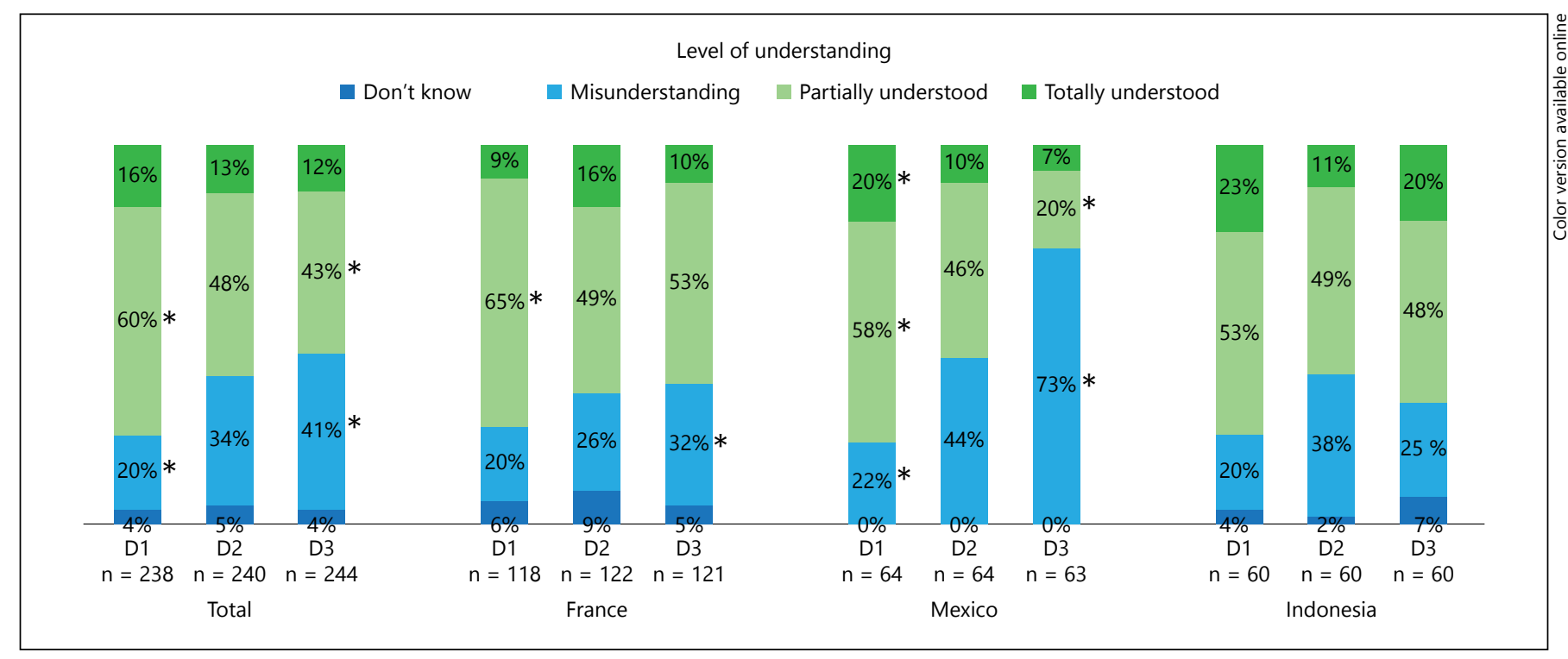

Fig. 2. The global level of understanding of 3 designs (D1, D2, and D3) of the urine color tool, in the total sample and according to country. ${ }^{*}$ p-value of t-test indicated a significant difference from the other 2 designs.

did. In Indonesia, the level of understanding of the 3 designs was comparable; whereas in both France (74\%) and Mexico (78\%), significantly more participants totally and partially understood D1 compared to the other 2 artworks.

Statistics regarding the understanding of the urine color demonstrated that $87 \%$ and $88 \%$ of the participants partially or totally understood the 3 light colors of the urine color chart on D1 and D2. The light colors were significantly less understood in design $\mathrm{D} 2$. However, $80 \%$ of the participants totally agreed with the phrase if my pee is light-coloured, it means I am drinking enough water' independently of the artwork. Dark colors were equally well understood. Again, significantly higher scores for total and partial understanding were observed with D1 and D3 compared with D2. Overall, 18\% of the participants misunderstood the message of D2 and reported incorrect interpretations such as 'I think I have an infection' or 'I do not pee enough.'

The level of attractiveness of the 3 designs was comparable, independent of country, age, and gender. On average, $80 \%$ of all participants liked the 3 designs a bit or a lot. Only $14 \%$ did not like the designs, and $5 \%$ of participants had no opinion regarding attractiveness.

When combining the results of the level of understanding and attractiveness, D1 obtained a significantly higher score compared to the other 2 designs (figure 3). D1 was totally or partially understood by $76 \%$ of the total participants from the 3 countries, and it was liked by $82 \%$ of them.

\section{Discussion and Conclusion}

The urine color scale was introduced and validated as an applicable tool for hydration assessment in adults [12], and recently also in children [13]. The latter clinical trial also reported that boys and girls of 8-14 years of age are able to accurately self-diagnose hypohydration based on their urine color [13]. The capability of the self-diagnosis is an important first step. In order for children to use the tool, they first of all have to find it 'fun' or 'cool.' However, during the field survey, the attractiveness of all 3 designs of phase 2 was equal. Therefore, the determining factor for the selection was the level of understanding of the tool. Three out of four children understood the message of the tool. The limitation of the survey is that the effectiveness of the tool to stimulate children to drink more water was not evaluated. This remains to be determined in an intervention study. Despite this limitation, several strengths of the hydration self-assessment tool are recognized. The tool (artwork D1, figures 1-3) is based on the urine color chart, a validated scale to assess human hydration status $[12,13]$. Moreover, the tool is developed with children for children, demonstrating that the tool is actually understood without further instructions and found attractive by children. This understanding was not linked to one nationality or language, in that the tool is illustration based and was evaluated by children from 3 different countries. Although encouraging, future research is required to 


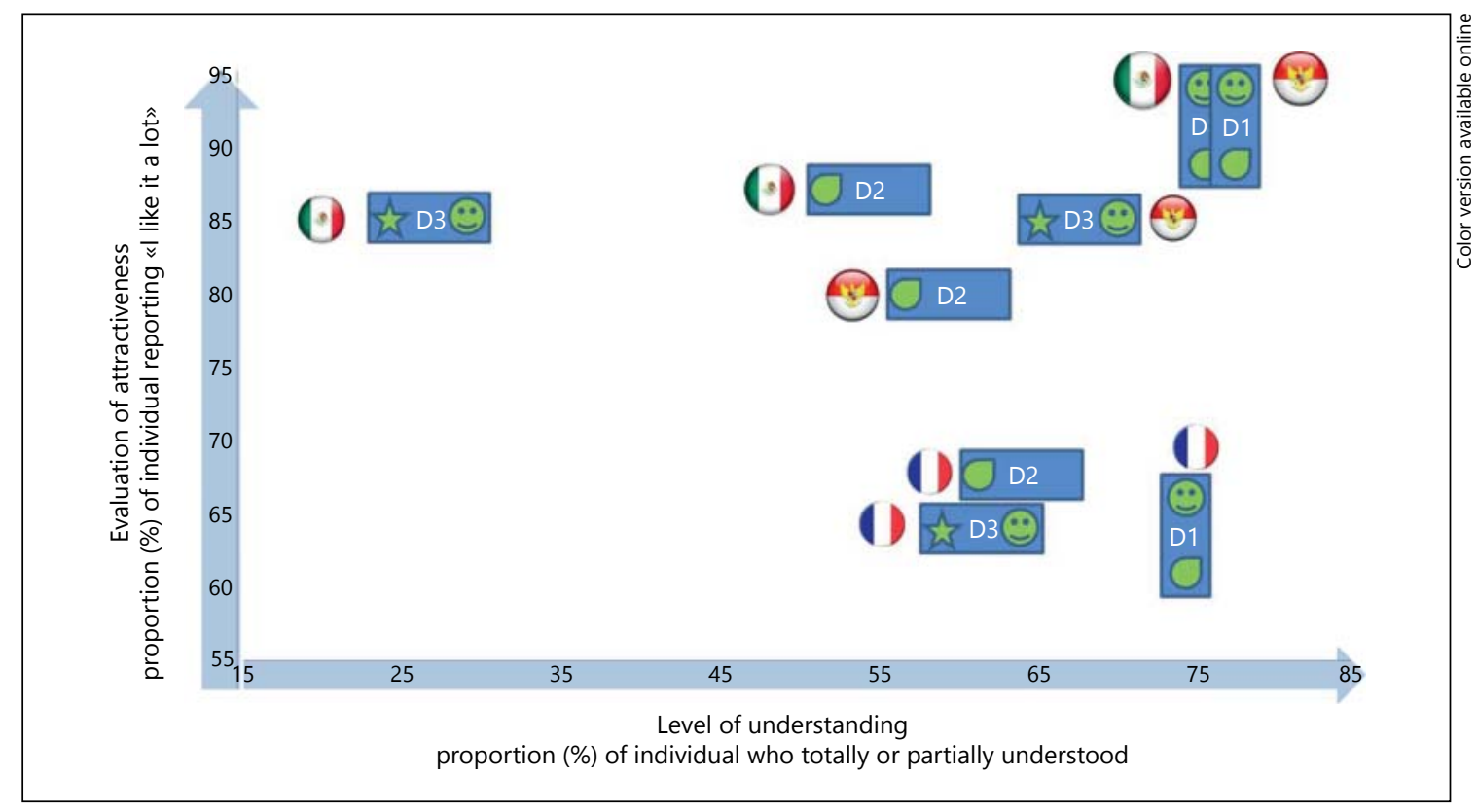

Fig. 3. Selection of the final artwork of the urine color tool by combining the level of understanding and attractiveness of the different chart designs in 3 countries.

determine whether this understanding exists in other countries and cultures worldwide.

We conclude that three out of four children liked the design and understood the correct hydration message from this strictly illustration-based tool containing the validated 8-category urine color scale.

\section{Disclosure Statement}

IG, ASM, and EE are employees of Danone Research. LEA and SAK are consulting members of the Scientific Advisory Board of the Hydration \& Health Department, Danone Research and have received research funding from Danone Research.

\section{Reference}

1 Altman PL: Blood and other body fluids Washington, DC, 1961.

2 Silvaggio T, Mattison DR: Comparative approach to toxicokinetics. In: Wilkins Wa, editor. Occupational and environmental reproductive hazards: a guide for clinicians. Baltimore, 1993, pp 25-36.

3 EFSA Panel on Dietetic Products Nutrition and Allergies (NDA). Scientific Opinion on Dietary reference values for water. EFSA Journal 2010;8:1459.

4 Iglesia-Altaba I, Guelinckx I, Salas-Salvado J, Kavouras S, Gandy J, Martinez H, et al: Total fluid intake of children and adolescents: cross sectional surveys in 13 countries worldwide. EJN 2015.

5 Bar-David Y: The effect of voluntary dehydration on cognitive functions of elementaty school children. Acta Paediatr 2005;94:16671673.

Assessing Hydration in Children
6 Dougherty KA, Baker LB, Chow M, Kenney WL: Two percent dehydration impairs and six percent carbohydrate drink improves boys' basketball skills. Med Sci Sports Exerc 2006;38:1650-1658.

7 Ebbeling CB, Feldman HA, Chomitz VR, Antonelli TA, Gortmaker SL, Osganian SK, et al: A randomized trial of sugar-sweetened beverages and adolescent body weight. $\mathrm{N}$ Engl J Med 2012;367:1407-1416.

8 Edmonds CJ, Jeffes B: Does having a drink help you think? 6-7-Year-old children show improvements in cognitive performance from baseline to test after having a drink of water. Appetite 2009;53:469-472.

-9 Kavouras SA, Arnaoutis G, Makrillos M, Garagouni $\mathrm{C}$, Nikolaou E, Chira O, et al: Educational intervention on water intake improves hydration status and enhances exercise performance in athletic youth. Scand J Med Sci Sports 2011;22:684-689.
10 Fiorito LM, Marini M, Mitchell DC, Smiciklas-Wright H, Birch LL: Girls' Early Sweetened Carbonated Beverage Intake Predicts Different Patterns of Beverage and Nutrient Intake across Childhood and Adolescence. J Am Diet Assoc 2010;110:543-550.

11 Armstrong LE: Assessing hydration status: the elusive gold standard. J Am Coll Nutr 2007;26(5 suppl):575S-584S.

12 Armstrong LE, Maresh CM, Castellani JW, Bergeron MF, Kenefick RW, LaGasse KE, et al: Urinary indices of hydration status. Int J Sport Nutr 1994;4:265-279.

13 Kavouras SA, Johnson EC, Bougatsas D, Arnaoutis G, Panagiotakos DB, Perrier E, et al: Validation of the urine color scale in children. European Journal of Nutrition 2015, in Press. 